\title{
Avaliação da debulha mecânica em sementes de milho ${ }^{1}$
}

\author{
Francisco de A. C. Almeida ${ }^{2}$, Maria E. C. de Oliveira ${ }^{3}$, Jogerson P. G. Pereira ${ }^{2}$, \\ Rubens F. da Costa ${ }^{4}$, Acácio Figueirêdo Neto ${ }^{3}$ \& Francisco M. de M. Oliveira ${ }^{3}$
}

\begin{abstract}
RESUMO
No estado da Paraíba, as perdas de sementes e grãos de milho (Zea mays L.) provocadas principalmente pela colheita, debulha mecânica, beneficiamento e processos subseqüentes, são da ordem de $20 \%$, acarretando prejuízos econômicos ao estado. Assim, estudaram-se as danificações decorrentes da debulha mecânica e da passagem das sementes de milho, variedades Cruzeta e Sertanejo, pelas diferentes etapas do beneficiamento. Para avaliar os danos provocados às sementes, utilizou-se uma debulhadora estacionária trabalhando a rotações de 520, 600 e 700 rpm, obtidas pela variação da aceleração do motor do trator ao qual a debulhadora se achava acoplada, como também das etapas promovidas pela UBS. Os danos foram avaliados visualmente e analisados em um delineamento estatístico inteiramente casualizado, com os tratamentos distribuídos em esquema fatorial. A variedade Cruzeta foi mais resistente aos impactos provocados pela debulha e pelo beneficiamento; o efeito do impacto na qualidade das sementes torna-se mais evidente com a elevação da velocidade do cilindro debulhador; a rotação de 600 rpm mostrou-se a mais indicada para a debulha do milho Cruzeta e Sertanejo.
\end{abstract}

Palavras-chave: debulhadora, danos mecânicos, sementes Zea mays

\section{Evaluation of mechanical thrashing processing in corn seeds}

\begin{abstract}
In the State of Paraiba, the loss of seeds and grains of corn (Zea mays L.) caused, mainly by harvesting, mechanical thrashing and subsequent processes is about $20 \%$ resulting in economic loss to the state. In this context, the effect of mechanical damages during the thrashing and passage of corn seeds varieties "Cruzeta" and "Sertanejo" were studied, in the different stages of processing. To evaluate the damages caused to the seeds, a stationary thrasher was used working at 520, 600 and $700 \mathrm{rpm}$, obtained by the acceleration variation of the tractor to which the thrasher was attached. The damages were visually appraised and analysed in a completely randomized statistical design with the treatments distributed in a factorial scheme. "Cruzeta" variety was more resistant to the impacts caused by the thrashing and processing; the impacts on the seed quality became more evident with the elevation of speed of the thrashing cylinder; the $600 \mathrm{rpm}$ rotation proved to be better for the thashing of Cruzeta and Sertanejo corn.
\end{abstract}

Key words: thrashing, mechanicals damages, Zea mays

1 Parte da Dissertação de Mestrado apresentada pelo segundo autor na UFCG

2 UAEAg/UFCG. Av. Aprígio Veloso, 882, CEP 58109-970, Campina Grande, PB. Fone: (83) 3310-1286. E-mail: almeida@deag.ufcg.edu.br

${ }^{3}$ Mestrado em Engenharia Agrícola, UFCG. Fone: (83) 3339-6060. E-mail: edcantic@bol.com.br

${ }^{4}$ EMEPA, CEP 58390-000, Alagoinha, PB. Fone: (83) 3278-1243. E-mail: rubens56@uol.com.br 


\section{INTRODUÇÃO}

No Brasil, a lavoura do milho tem-se expandido nos últimos anos, a ponto de produzir $7,1 \%$ de toda a produção mundial que, na safra de 2001/2002, foi da ordem de 593,39 milhões de toneladas (Corretora Mercado, 2003). Segundo Franceschini et al. (1996) dentro da produção nacional de cereais e leguminosas o desempenho da lavoura de milho exerce efeito direto e significativo sobre o volume da colheita: de cada três quilogramas colhidos, mais de um vem do milho e o que era considerado lavoura tipicamente de subsistência, passou a ser lavoura de interesse comercial.

$\mathrm{Na}$ operação de colheita do milho empregam-se os sistemas manual e mecanizado, sendo que, na Paraíba é trabalhado, em quase sua totalidade, o sistema semimecanizado, que consiste na colheita manual das espigas, as quais são depois submetidas ao processo de debulha em máquinas de pequeno porte, geralmente acopladas ao sistema hidráulico do trator e acionadas pelo eixo da tomada de potência do mesmo. A mecanização é utilizada em todas as fases de produção de sementes, razão por que os equipamentos devem ser regulados de maneira a evitar perdas no momento da colheita. Segundo Vasconcelos et al. (2002) o percentual de perda aceitável na colheita está em torno de $4 \%$.

No Brasil, a colheita manual é de 54,3\% aproximadamente e apresenta perda média de 1 a 1,5\% enquanto a colheita mecanizada corresponde a $45,7 \%$ da produção, podendo ocorrer perdas de 8 a $10 \%$.

Visando à redução dessas perdas, pesquisas têm sido realizadas buscando-se desenvolver novos projetos de máquinas de processamento e beneficiamento, para melhorar a eficiência das operações, de acordo com as características de cada cultura, desde o campo até o galpão pré-industrial. Dentre essas pesquisas estuda-se o milho em que a debulha, manual ou mecânica, danifica as sementes e, por isso, é considerado um dos fatores responsáveis pela redução da qualidade das sementes; na colheita mecânica é imprescindível conhecer a relação entre a velocidade de rotação do cilindro debulhador e o teor de água da semente, no momento da debulha.

De acordo com Ruffato et al. (2001) quanto mais seco o grão estiver menor será a elasticidade, tornando-o vulnerável aos danos provenientes da ação dos equipamentos. Segundo Andrade et al. (1998) os danos mecânicos em sementes durante a colheita, a debulha e o beneficiamento, são extremamente prejudiciais à qualidade das sementes, pois reduz 0 valor do produto que sofreu injúria, tem seu valor de mercado reduzido, até mesmo por seu aspecto visual. Todas as etapas do processo produtivo têm conseqüências em virtude dos golpes aplicados sobre as sementes, com a finalidade de separá-las da estrutura que as contém (Andrade et al., 1999). A injúria mecânica é considerada, por muitos tecnologistas de sementes, um dos mais sérios problemas da produção de sementes cuja principal causa é a mecanização nas várias fases dos processos: colheita, transportes, secagem e beneficiamento (Paiva, 1997).

Os estudos sobre efeitos de danificação em sementes de milho na região Nordeste do Brasil, especialmente na Paraí- ba, são bastante escassos, bem como a existência de dados sobre teores de umidade mais favoráveis para a colheita e debulha das espigas.

Por esses motivos e devido ao incremento na utilização de debulhadoras mecânicas em colheitas semi-mecanizadas no Estado, estudaram-se alguns pontos relativos às danificações mecânicas em sementes de milho, visando reduzir a gravidade de seus efeitos, onde se preconiza, como objetivo, verificar o comportamento das sementes de milho das variedades Cruzeta e Sertanejo sob a ação de diferentes rotações do cilindro debulhador da máquina e o beneficiamento em uma UBS.

\section{MATERIAL E MÉTODOS}

O trabalho foi conduzido no Laboratório de Armazenamento e Pré-Processamento de Produtos Agrícolas (LAPPA) da Unidade Acadêmica de Engenharia Agrícola (UAEAg) da Universidade Federal de Campina Grande (UFCG) e na Unidade de Beneficiamento de Sementes (UBS) da Empresa Estadual de Pesquisa Agropecuária do Estado da Paraíba (EMEPA) localizada no município de Alagoinha, PB, com Latitude $6^{\circ} 57^{\prime} 32^{\prime \prime} \mathrm{S}$, Longitude $35^{\circ} 33^{\prime} 9^{\prime \prime} \mathrm{W}$ e altitude de $113 \mathrm{~m}$.

Utilizaram-se sementes de milho de duas variedades, Cruzeta e Sertanejo, produzidas na safra 2001/2002, procedentes de campo experimental da EMEPA e que tinham $10 \%$ b.u. de água, por ocasião da debulha.

Do material colhido retirou-se uma amostra de cada variedade para debulha manual, referente à testemunha. As rotações utilizadas para a debulha mecânica foram 520, 600 e 700 rpm; a seguir, as sementes foram beneficiadas passando por todas as etapas da UBS (pré-limpeza, mesa de ar e peneira, elevador vibratório, mesa de gravidade e pesagem), obedecendo ao esquema operacional da Figura 1; em cada etapa e para cada rotação do cilindro debulhador tomaram-se, depois da análise de pureza, $3 \mathrm{~kg}$ de sementes para avaliação dos danos mecânicos.

As velocidades do cilindro, da debulhadora estacionária, modelo PAIN, $\mathrm{n}^{\circ} 315$, acoplada a um trator Massey Ferguson 4 x 2, pneus de borracha, com acionamento rotativo na TDP, foram determinadas por tacômetro modelo Venture, com leitura máxima de $50.000 \mathrm{rpm}$.

As sementes de cada tratamento foram submetidas aos seguintes testes:

\section{Pureza física}

Realizada de acordo com as Regras para Análise de Sementes (Brasil, 1992) exceto quanto a quantidade de sementes, que foi de $10 \mathrm{~kg}$ para cada rotação e, para o cálculo das impurezas (\%), pesou-se todo o material inerte. A porcentagem da pureza (Eq. 1) foi determinada pela relação entre a massa das sementes puras e a massa total da amostra.

$$
\mathrm{Pz}=100 \quad 1 \frac{\mathrm{m}_{\mathrm{i}}}{\mathrm{m}_{\mathrm{m}}}
$$




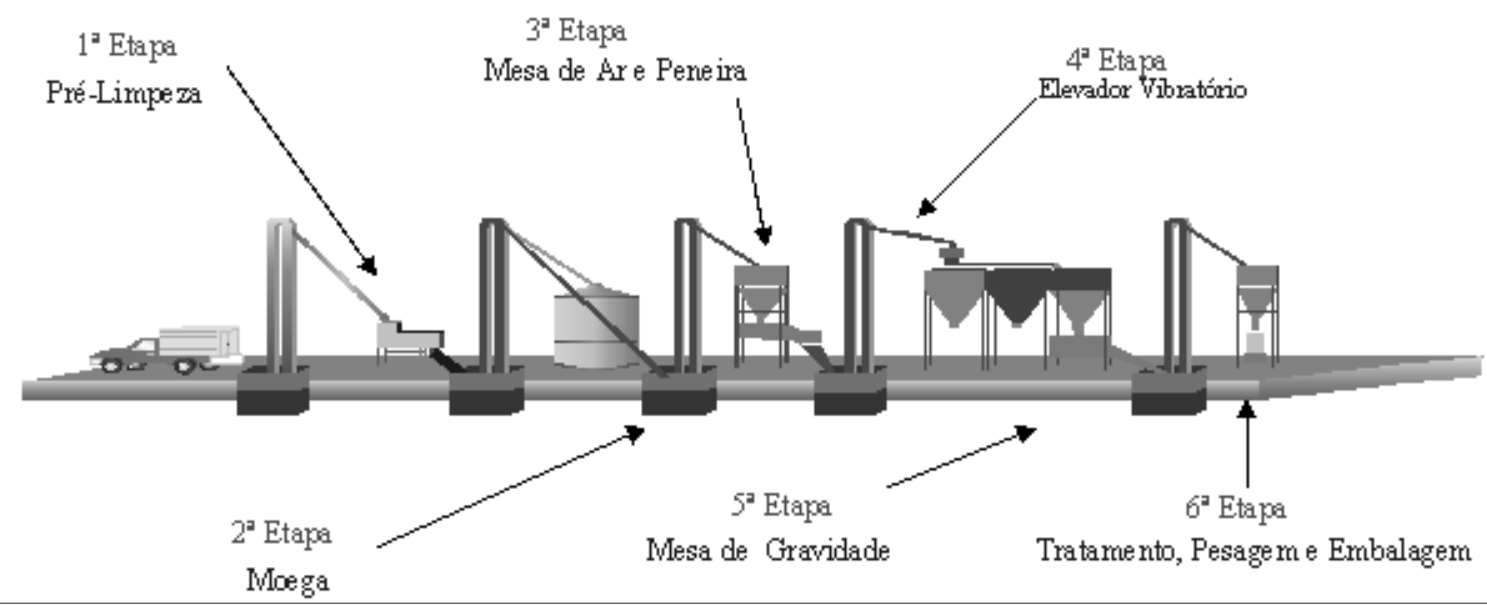

Figura 1. Esquema operacional da Unidade de Beneficiamento de Sementes (UBS)

em que:

$\mathrm{P}_{\mathrm{z}}$ - pureza física de sementes, $\%$

$\mathrm{m}_{\mathrm{i}}$ - massa de impureza, $\mathrm{g}$

$\mathrm{m}_{\mathrm{m}}$ - massa total da amostra, $\mathrm{g}$

\section{Teor de água}

O controle da umidade das sementes no campo foi efetuado pelo método da estufa, a $130 \pm 2{ }^{\circ} \mathrm{C}$, durante $4 \mathrm{~h}$, obedecendo às Regras para Análise de Sementes (Brasil, 1992) utilizando-se três sub-amostras de $30 \mathrm{~g}$, em cada repetição. Por ocasião da debulha empregou-se o método-padrão da estufa a $105 \pm 3{ }^{\circ} \mathrm{C}$, durante $24 \mathrm{~h}$, para conhecimento do teor de água (Brasil, 1992).

\section{Danos mecânicos}

Logo depois da debulha em cada rotação, os danos foram avaliados visualmente, utilizando-se uma amostra de $800 \mathrm{~g}$, distribuídos em quatro repetições de $200 \mathrm{~g}$ cada uma; nessa determinação se seguiu a recomendação de Andrade et al. (1999) usando uma bancada com fundo branco para promover melhor identificação das sementes. Os danos mecânicos foram classificados nas três categorias:

a. danos leves: sementes com tegumento rompido ou com fissura

b. danos intermediários: sementes visualmente com trinca (partidas ao meio internamente, protegidas apenas pelo pericarpo)

c. danos severos: sementes partidas ao meio

O cálculo do índice de danos mecânico foi obtido através da expressão:

$$
\mathrm{D}=100 \frac{\mathrm{m}_{\mathrm{d}}}{\mathrm{m}_{\mathrm{m}}}
$$

em que:

D - índice de danos mecânicos, \%

$\mathrm{m}_{\mathrm{d}}$ - massa de sementes com danos mecânicos, $\mathrm{g}$

$\mathrm{m}_{\mathrm{m}}$ - massa total da amostra, $\mathrm{g}$

\section{Delineamento estatístico}

Realizou-se a análise dos dados com o auxílio do progra- ma computacional Assistat versão 6,5 beta (Silva \& Azevedo, 2002). As médias dos tratamentos foram comparadas pelo teste de Tukey a 1 e $5 \%$ de probabilidade. Os danos mecânicos ocorridos no momento da debulha e no beneficiamento das sementes, foram analisados em esquema fatorial $2 \times 3$, sendo duas variedades (Sertanejo e Cruzeta) e três rotações do cilindro debulador $(520,600,700 \mathrm{rpm})$ em um delineamento estatístico inteiramente casualizado, com três repetições.

\section{RESULTADOS E DISCUSSÃO}

Examinando-se a Tabela 1, verifica-se que na debulha as médias encontradas na rotação $R_{3}(700 \mathrm{rpm})$ foram superiores a $R_{1}$ e $R_{2}$, tanto para a variedade Sertanejo como para a variedade Cruzeta; comportamento semelhante se constata com o beneficiamento no qual $R_{2}$ e $R_{3}$ superaram $R_{1}$ e não diferiram estatisticamente entre si.

A comparação entre variedades indica superioridade da Sertanejo sobre a Cruzeta nos dois processos (debulha e beneficiamento).

Tabela 1. Valores médios* $(\%)$ de pureza física de sementes de milho debulhadas sob ação da rotação do cilindro debulhador e beneficiadas na UBS

\begin{tabular}{|c|c|c|c|}
\hline \multirow{2}{*}{ Variedade } & \multicolumn{3}{|c|}{$\begin{array}{c}\text { Pureza física na debulha } \\
\text { Rotações }\end{array}$} \\
\hline & $R 1=520$ & $R 2=600$ & $\mathrm{R} 3=700$ \\
\hline Sertanejo & $91,86 \mathrm{aB}$ & $92,34 \mathrm{aB}$ & $93,87 \mathrm{aA}$ \\
\hline Cruzeta & 89,52 bB & 87,64 bC & $90,63 \mathrm{bA}$ \\
\hline \multirow{2}{*}{ DMS } & Entre linha & & 0,58 \\
\hline & Entre coluna & & 0,47 \\
\hline CV (\%) & & & 0,35 \\
\hline \multicolumn{4}{|c|}{ Pureza física no beneficiamento } \\
\hline Sertanejo & $96,94 \mathrm{aB}$ & 97,58 aA & 97,52 aA \\
\hline Cruzeta & 94,42 bB & 96,11 bA & $96,51 \mathrm{bA}$ \\
\hline \multirow{2}{*}{ DMS } & Entre linha & & 0,41 \\
\hline & Entre coluna & & 0,34 \\
\hline CV (\%) & & & 0,23 \\
\hline
\end{tabular}

*Médias seguidas pelas mesmas letras maiúsculas na linha e minúsculas na coluna, não diferem entre si pelo teste de Tukey, a 5\% de probabilidade 
Confrontando-se as médias dos dois processos, vê-se que a pureza física após o beneficiamento foi superior $(5,54 \%)$ à pureza física da debulha. Rocha et al. (1994) constataram que as sementes de milho saíram da máquina de pré-limpeza (início do processo) com $14,8 \%$ de impurezas e deixou a balança ensacadora (final do processo de beneficiamento) com 9,2\% de impurezas, concluindo, então, que houve redução significativa das impurezas sempre que o produto foi submetido a passagem pelos equipamentos, durante o processo de beneficiamento.

No presente trabalho, a colheita foi manual; logo, as impurezas, como fragmentos de sementes, trincas, frações de sabugo e palhas, resultaram da máquina debulhadora e da passagem das sementes pelas etapas do beneficiamento na UBS; esses resultados indicam a necessidade de se ter informações sobre a qualidade das máquinas, em termos de adaptabilidade à carência do usuário e que a falta de testes e ensaios de máquinas agrícolas dificulta a vida do produtor, que conta com pouca segurança na hora de comprar seu maquinário.

Como se observa pelos resultados, as duas variedades de milho (Sertanejo e Cruzeta) atendem a classificação quanto à qualidade para impurezas e materiais estranhos, requerida pelas normas de classificação do milho (Puzzi, 2000) em que permite como tolerância máxima, 1,5; 2,0 e 3,0\% para os tipos 1, 2 e 3, respectivamente. Brooker et al. (1992) descreveram que, dentre os principais fatores que determinam a qualidade dos grãos e sementes, está a pureza física; desta forma, as máquinas de debulha que proporcionam elevado percentual de pureza são preferidas, pois diminuem a necessidade de processamento posterior para limpeza do produto.

Os resultados referentes à avaliação do grau de danificação em sementes de milho das variedades Sertanejo e Cruzeta, debulhado mecanicamente indicam, conforme dados da Tabela 2, maior resistência aos impactos impostos pela máquina debulhadora da variedade Cruzeta $(1,22)$ frente à variedade Sertanejo $(3,02)$ em 59,6\%. Verifica-se, ainda, que a debulha realizada a uma rotação de $600 \mathrm{rpm}$ promoveu menor índice de danos mecânicos e este foi maior quando a rotação foi de $700 \mathrm{rpm}$ enquanto a debulha na rotação de $520 \mathrm{rpm}$ danificou mais as sementes que a $600 \mathrm{rpm}$ e menos que a 700 rpm.

Tabela 2. Valores médios* (\%) de sementes de milho das variedades Sertanejo e Cruzeta danificadas pelos impactos ocorridos durante o processo de debulha, para a interação variedades $x$ rotações

\begin{tabular}{llll}
\hline \multirow{2}{*}{ Variedade } & \multicolumn{3}{c}{ Rotações } \\
\cline { 2 - 4 } & $\mathbf{R}_{\mathbf{1}}=\mathbf{5 2 0}$ & $\mathbf{R}_{\mathbf{2}}=\mathbf{6 0 0}$ & $\mathbf{R}_{\mathbf{3}}=\mathbf{7 0 0}$ \\
Sertanejo & $3,56 \mathrm{aA}$ & $2,46 \mathrm{aC}$ & $3,06 \mathrm{aB}$ \\
Cruzeta & $1,30 \mathrm{bAB}$ & $0,93 \mathrm{bB}$ & $1,43 \mathrm{bA}$ \\
\multicolumn{1}{c}{ D.M.S. } & Entre linha & & 0,32 \\
CV (\%) & Entre coluna & & 0,39 \\
\hline
\end{tabular}

* Médias seguidas pelas mesmas letras maiúsculas na linha e minúsculas na coluna não diferem entre si, pelo teste de Tukey, a $5 \%$ de probabilidade
Borba et al. (1992) constataram, na debulha mecânica de milho colhido manualmente, com 10, 15,5 e 22\% de água, que as rotações de 400 e $700 \mathrm{rpm}$ com umidade de 10 e $22 \%$ de água provocaram danos mecânicos da ordem de 7,4 e 54,2\%, respectivamente. Sobre o tema, Pedroza (1991) também verificou que os valores do índice de danos em sementes de milho da variedade Central Mex, cresceram com o aumento da velocidade do cilindro debulhador.

Souza et al. (2002) relataram que a presença de danos mecânicos em sementes colhidas e debulhadas mecanicamente é inevitável e isto, além de prejudicar a qualidade da semente para o plantio, afeta também a qualidade dos grãos, diminuindo consideravelmente o seu valor de mercado para o consumo; portanto, os danos mecânicos podem ser altamente prejudiciais ao produto final, seja ele destinado ao consumo ou ao plantio, tema apontado por Paiva et al. (2000) como ponto de estrangulamento no processo de produção de sementes de alto padrão de qualidade.

A Tabela 3 contém os resultados obtidos na avaliação visual das danificações mecânicas ocorridas durante o beneficiamento na UBS. Observa-se que a intensidade de danos

Tabela 3. Valores médios* $(\%)$ de sementes de milho das variedades Sertanejo e Cruzeta danificadas pelos impactos ocorridos durante o processo de beneficiamento na UBS para a interação variedades $x$ rotações

\begin{tabular}{llll}
\hline Variedade & \multicolumn{3}{c}{ Rotações } \\
\cline { 2 - 4 } & $\mathbf{R}_{\mathbf{1}}=\mathbf{5 2 0}$ & $\mathbf{R}_{\mathbf{2}}=\mathbf{6 0 0}$ & $\mathbf{R}_{\mathbf{3}}=\mathbf{7 0 0}$ \\
Sertanejo & $1,23 \mathrm{aB}$ & $1,56 \mathrm{aA}$ & $0,83 \mathrm{aC}$ \\
Cruzeta & $0,56 \mathrm{bB}$ & $0,50 \mathrm{bB}$ & $1,03 \mathrm{aA}$ \\
\multicolumn{1}{c}{ D.M.S. } & Entre linha & & 0,21 \\
CV (\%) & Entre coluna & & 0,26 \\
\hline
\end{tabular}
entre si pelo teste de Tukey, a $5 \%$ de probabilidade

ocorridos na variedade Sertanejo foi superior $(1,20)$ aos resultados apresentados pela variedade Cruzeta $(0,69)$ em 42,5\%. Notou-se, entre as rotações, que a de $600 \mathrm{rpm}\left(\mathrm{R}_{2}\right)$ na variedade Sertanejo obteve o maior percentual de danos. As sementes foram mais danificadas na variedade Cruzeta, com rotação do cilindro debulhador de $700 \mathrm{rpm}\left(\mathrm{R}_{3}\right)$ seguida pelas rotações $520 \mathrm{rpm}\left(\mathrm{R}_{1}\right)$ e $600 \mathrm{rpm}\left(\mathrm{R}_{2}\right)$ cujo fato se deve, provavelmente, às sementes trincadas provenientes do processo de debulha que se manifestaram posteriormente pelos impactos provocados pelo equipamento da UBS. Sobre o tema, Lersch Júnior (1999) comenta que os danos mecânicos são produzidos progressivamente, a partir da debulha mecânica e no final do beneficiamento. De acordo com Ruffato et al. (2001) a susceptibilidade à quebra do produto, nas operações subseqüentes, aumenta com a intensidade e o número de trincas presentes, reduzindo a qualidade dos grãos em vários aspectos. 


\section{CONCLUSÕES}

1. Os maiores percentuais de quebras nas sementes (danos) ocorreram nas rotações de 520 e $700 \mathrm{rpm}$.

2. A variedade Cruzeta foi mais resistente à quebra na debulha e no beneficiamento que a variedade Sertaneja.

3. Houve efeitos imediatos de danificações mecânicas provocadas pela debulha e beneficiamento.

4. A rotação de $600 \mathrm{rpm}$ é a mais indicada para a debulha das sementes do milho Cruzeta e Sertanejo com 10\% de umidade (b.u.).

5. O beneficiamento reduziu o percentual de impurezas promovido pela debulha mecânica.

6. As impurezas diminuem com a elevação da rotação do cilindro debulhador.

\section{LITERATURA CITADA}

Andrade, E.T. de; Corrêa, P.C.; Alvarenga, E.M.; Martins, J.H. Efeitos de danos mecânicos controlados sobre a qualidade fisiológica de sementes de feijão durante o armazenamento. Revista Brasileira de Armazenamento, Viçosa, v.23, n.2, p.41-51, 1998.

Andrade, E.T. de; Corrêa, P.C.; Martins, J.H.; Alvarenga, E.M. Avaliação de dano mecânico em sementes de feijão por meio de condutividade elétrica. Revista Brasileira de Engenharia Agrícola e Ambiental, Campina Grande, v.3, n.1, p.54 $-60,1999$.

Borba, C.S.; Andrade, R.V.; Azevedo, J.T.; Oliveira, A.C. Debulha mecânica, teor de umidade e qualidade das sementes. Centro Nacional de Pesquisa de Milho e Sorgo. Sete Lagoas: Embrapa-CNPMS, 1992, p.204-205. Relatório Técnico Anual 1988-1991.

Brasil. Ministério da Agricultura e Reforma Agrária. Regras para análise de sementes. Brasília: SNDA/DNDV/CLAV, 1992. 365p.

Brooker, D.B.; Bakkel-Arkema, F.W.; Hall, C.M. Drying and storage of grains and oil seeds. New York: van Nostrand Reinhold. 1992. 420p.

Corretora Mercado. Mercantil e Corretora de Mercadorias. http:// www.corretoramercado.com.br/estatistica. 06 Mai. 2003.
Franceschini, A.S.; Martins, J.H.; Correa, P.C.; Faroni, L.R.D.A.; Cecon, P.R. Avaliação da qualidade do milho BR201 submetido a diferentes condições de secagem. Revista Brasileira de Armazenamento, Viçosa, v.21, n.1/2, p.13-16, 1996.

Lersch Jr., I. Qualidade das sementes nas etapas de beneficiamento do milho. Resumo de tese/dissertação, Universidade Federal de Santa Maria, RS, 1999. http://ged.capes.gov.br/AgTest/silverstream/pages/pgRelatorioTesesCompleto.html?ANOBA. 07 Abr. 2003.

Paiva, L.E. Danos mecânicos em sementes de milho Ag-122 no beneficiamento, colhidas mecanicamente em espigas e em grãos, e seu comportamento no armazenamento e desempenho em campo. Lavras: UFLA, 1997. 102p. Tese Doutorado

Paiva, L.E.; Medeiros Filhos, S.; Fraga, A.C. Beneficiamento de sementes de milho colhidas mecanicamente em espigas: Efeitos sobre danos mecânicos e qualidade fisiológica. Ciência Agrotécnica, Lavras, v.24, n.4, p.846-856, 2000.

Pedroza, J.P. Efeito de danos mecânicos sobre propriedades físicas de grãos de milho. Campina Grande: UFPB. 1991. 72p. Dissertação Mestrado

Puzzi, D. Abastecimento e armazenamento de grãos. Campinas: Instituto Campineiro de Ensino Agrícola, 2000, 666p.

Rocha, F.E.C.; Silva, E.C. de; Alves, E.R. de S.; Teixeira, R.N. Danos em sementes de milho durante beneficiamento. Pesquisa Agropecuária Brasileira, Brasília, v.29, n.8, p.12811285, 1994.

Ruffato, S.; Couto, S.M.; Queiroz, D.M. Análise de impactos em grãos de milho pelo método de elementos finitos. Revista Brasileira de Armazenamento, Viçosa, v.26, n.1, p.21-27, 2001.

Silva, F.A.S. e; Azevedo, C.A.V. de. Versão do programa computacional Assistat para o sistema operacional for windows. Revista Brasileira de Produtos Agroindustriais, Campina Grande, v.4, n.1, p.71-78, 2002.

Souza, C.M.A.; Queiroz, D.M.; Mantovani, E.C.; Cecon, P.R. Efeito da colheita mecanizada sobre a qualidade de sementes de feijão (Phaseolus vulgaris L.). Revista Brasileira de Armazenamento, Viçosa, v.27, n.1, p.21-29, 2002.

Vasconcelos, R.C.; von Pinho, R.G.; Reis, R.P.; Logato, E.S. Tecnologias aplicadas na cultura do milho em Lavras, MG na safra 1998/1999. Ciência Agrotécnica, Lavras, v.26, n.1, p.117-127, 2002. 